\title{
A Numerical Investigation on Nonparametric Identification of Nonlinear Roll Damping Moment of a Ship from Transient Response
}

\author{
T. S. Jang ${ }^{*}, 1$ J.W. Son ${ }^{2}$, S.L. Han ${ }^{1}$, H.G. Sung ${ }^{3}$, S.K. Lee ${ }^{1}$ and S.C. Shin ${ }^{1}$ \\ ${ }^{I}$ Department of Naval Architecture and Ocean Engineering, Pusan National University, Busan 609-735, South Korea \\ ${ }^{2}$ Sungdong Shipbuilding and Marine Engineering Co., Ltd, Hwang-ri, Gwangdo-myeon, Tongyeong-si, Gyeongsang- \\ nam-do 650-827, South Korea \\ ${ }^{3}$ Maritime and Ocean Engineering Research Institute, KORDI, 171 Jang-dong, Yusung-gu, Daejeon 305-343, South \\ Korea
}

\begin{abstract}
This paper is concerned with the determination of nonlinear roll damping moments of ships including similar floating structures. Using the measured motion response data of ship's roll motion, an inverse problem is formulated for the determination. The problem of damping determination is mathematically involved in a Volterra integral equation of the "first" kind between the roll responses and unknown nonlinear roll damping. This first kind integral equation results in numerical instability, that is, solutions lack stability property. A regularization method is applied in order to suppress the instability. Any priori information is not required on the model of the nonlinear damping. The applicability of the present study is demonstrated through the numerical investigations using the given nonlinear roll equations from the tests.
\end{abstract}

Keywords: Nonlinear roll damping moment, transient response data, identification.

\section{INTRODUCTION}

It is well known that a highly nonlinear characteristic is strongly involved in the roll motion of ships, barges and similar floating structures. The reason for this is mainly because of a highly nonlinear characteristic in ship's complex damping mechanism such as the effect of fluid viscosity. The more important thing is that the dynamic stability of ships in realistic sea is dependent on its rolling motion. Thus, the investigation of ship's roll dynamics is most crucial unlike other degrees of freedom of ship motion. For the purpose, it is generally required to find ship's roll damping for accurate prediction of its response to various loading environments and development of control strategies: this is essential for the design of ship-shaped structures. However, the determining of the roll damping is difficult because of its strong nonlinearity.

A number of methods of determining nonlinear damping have been proposed in last decades. The works of the proposed methods have been usually concerned with nonlinear damping model through parameters based on intuition. Various assumed models for the nonlinear roll motion in damping can be found in the works [1-9]. For examples, Taylan $[4,5]$ and Wellicome [8] have utilized an assumed nonlinear damping model, expressed as $a \dot{\phi}+b \dot{\phi}^{2}$, while Odabasi and Vince [9] have used other form of damping model of $a \dot{\phi}+b \phi^{2} \dot{\phi}$. A cubic nonlinearity in damping, $a \dot{\phi}+b \dot{\phi}^{3}$, was also considered as a damping model type by Wright and

*Address correspondence to this author at the Department of Naval Architecture and Ocean Engineering, Pusan National University, Busan 609-735, South Korea; Tel.: +82.51.510.2789; Fax: +82.51.581.3718;

E-mail: taek@pusan.ac.kr
Marshfield [1] in their earlier works. However, according to Taylan $[4,5]$, the validity of the assumed damping models appears to be questionable in people's minds. Thus, it is needed to develop a new identification method for improving the validity of the damping models to achieve the accurate estimation of nonlinear roll damping of ships.

In this spirit, Jang et al. [10] introduced a new method, based on an inverse formulism that often occurs in many branches of science and engineering, to recover nonlinear damping in nonlinear oscillation systems. While, Jang et al. [11], based on an experiment of free-roll decay test for a ship, utilized the method [10] to recover the functional form of the nonlinear roll damping, the present paper is concerned with an application of the method $[10,11]$ to identify the nonlinear roll damping moment of a ship with the (known) data from the tests $[1,12-15]$. For the purpose, we first construct a functional relationship between the roll responses and unknown nonlinear roll damping as a Volterra integral equation [10]. On the basis of the constructed formulation, a procedure for identifying the functional form of the nonlinear roll damping moment for floating structures is described in this paper. The present study has particular advantages and remarkable improvements. First, it does not require any priori information on the model of the nonlinear damping. Second, this study directly uses transient response data to identify nonlinear damping moment unlike the earlier works utilizing roll decrement data. To verify the applicability of the method, we perform the identification of the nonlinear roll damping following the suggested procedure [10], in which we apply the nonlinear roll equations of a ship with the known data from tests $[1,12-15]$ to the procedure. We 
succeed in recovering the nonlinear roll damping moment of a ship in an accurate and stable manner.

\section{ROLL MOTION'S EQUATION}

In the case of ship roll motion, the roll angle $\phi$ is governed by a nonlinear ordinary differential equation of motion in the form:

$$
I \ddot{\phi}+c(\dot{\phi})+k(\phi)=0
$$

where $I$ denotes the total roll inertia and $c(\dot{\phi}), k(\phi)$ are the nonlinear damping and restoring functions, respectively. The nonlinear restoring function usually has the form as odd series in $\phi[16]$ :

$$
k(\phi)=\sum_{j=1,3, \ldots} k_{j} \phi^{j}=k_{1} \phi+\tilde{k}(\phi)
$$

where $\tilde{k}$ the nonlinear part for the restoring function is represented. With Eq. (2), Eq.(1) can be rewritten as

$$
I \ddot{\phi}+c(\dot{\phi})+k_{1} \phi+\tilde{k}(\phi)=0
$$

For the purpose of the identification of the nonlinear damping function $c(\dot{\phi})$, the differential equation for the nonlinear roll motion in Eq. (3) is transformed into an equivalent nonlinear integral equation: it is generally known that integral operators are stable, whereas differential operators are usually unstable $[10,11]$. From the concept of variation of parameters or variation of constants $[10,11,17]$, Eq. (3) can be transformed to following nonlinear Volterra integral equation as follows

$$
\phi(t)=\alpha \phi_{1}(t)+\beta \phi_{2}(t)-\int_{0}^{t} \frac{\phi_{1}(\tau) \phi_{2}(t)-\phi_{1}(t) \phi_{2}(\tau)}{I W(\tau)}[c(\dot{\phi}(\tau))+\tilde{k}(\phi(\tau))] d \tau .
$$

with the initial conditions

$\phi(0)=\alpha, \quad \dot{\phi}(0)=\beta$.

In Eq. (4), $\phi_{1}(t)$ and $\phi_{2}(t)$ are chosen to satisfy the following initial value problems $[10,11]$

$$
\begin{array}{ll}
I \ddot{\phi}_{1}+k_{1} \phi_{1}=0, & \phi_{1}(0)=1, \quad \dot{\phi}_{1}(0)=0 \\
I \ddot{\phi}_{2}+k_{1} \phi_{2}=0, & \phi_{2}(0)=0, \quad \dot{\phi}_{2}(0)=1
\end{array}
$$

and the Wronskian $W$ is defined as

$$
W(t) \equiv\left|\begin{array}{ll}
\phi_{1}(t) & \phi_{2}(t) \\
\dot{\phi}_{1}(t) & \dot{\phi}_{2}(t)
\end{array}\right| .
$$

\section{IDENTIFICATION PROCEDURE FOR THE NON- LINEAR DAMPING}

If the restoring function $k(\phi)$ is known a priori and the (transient) roll angle $\phi(t)$ and angular velocity $\dot{\phi}(t)$ are measured during the roll motion with the initial conditions in Eq. (5), then Eq. (4) becomes an Volterra type integral equation of the first kind for $x(t)$ in Eq. (8), because the lefthand-side of Eq. (8) can be known using the initial conditions $\alpha, \beta$ in Eq. (5) and $\phi_{1}(t), \phi_{2}(t)$ which satisfy Eq. (6) $[10,11,17,18]$ :

$$
y(t)=\int_{0}^{t} K(t, \tau) x(\tau) d \tau
$$

where $x, y$ and $K$ are defined respectively as follows,

$$
\begin{gathered}
x(t)=-c(\dot{\phi}(t)) \\
y(t)=\phi(t)-\alpha \phi_{1}(t)-\beta \phi_{2}(t)+\int_{0}^{t} \frac{\phi_{1}(\tau) \phi_{2}(t)-\phi_{1}(t) \phi_{2}(\tau)}{I W(\tau)}[\tilde{k}(\phi(\tau))] d \tau \\
K(t, \tau)=\frac{\phi_{1}(\tau) \phi_{2}(t)-\phi_{1}(t) \phi_{2}(\tau)}{I W(\tau)}
\end{gathered}
$$

Eq. (8) appears to be a Volterra type of nonlinear integral equation of the first kind for $x$. From theory of first kind integral equations, it is known that the first kind integral equation (8) having the regular kernel such as $K$ in Eq. (11) turns out to be ill-posed in the sense of stability [19]. The solution is concerned with numerical instability which will affect the performance of the present nonlinear damping identification. Unfortunately, the introduction of direct numerical methods only produces a solution with an arbitrarily large error because of the lack of stability (or ill-posedness). In fact, a direct discretization of the right hand side of Eq. (8) results in a matrix in which the condition number is extremely large so that the numerical inverse of the matrix does not work because the determinant of the matrix is nearly zero $[10,18,20-26]$. In order to overcome the difficulty of the numerical instability, a regularization method (i.e., stabilization technique) is introduced. A regularization is known as a powerful method to solve the unwelcome unstable numerical difficulty: in fact, it is a stabilization technique which suppresses the lack of stability (or instability) by adding an artificial stability property to the present problem.

The solution $x(t)$ to the integral equation (8) can be obtained by the following iteration, known as Landweber's regularization [10, 11, 18, 20-23, 27],

$$
x_{m}=\left(I-\lambda L^{*} L\right) x_{m-1}+\lambda L^{*} y, \quad m=1,2 \ldots,
$$

for a real positive constant $\lambda$ such that $0<\lambda<1 /\|L\|_{2}^{2}$, where $\|\cdot\|_{2}$ refers to the $L_{2}$ norm [19]. In Eq. (12), the operator $L$ is defined as follows, for an arbitrary function $b(t)$,

$$
L b=\int_{0}^{t} K(t, \tau) b(\tau) d \tau
$$

and $L^{*}$ represents the adjoint operator of $L$ and $I$ represents the identity operator [19]. Because the iteration converges to the solution for an arbitrary initial guess $x_{0}$, for convenience, we start with the initial guess of zero function in this paper $[10,11,18,20-26]$ :

$$
x_{0}=0
$$

With the (obtained) $x$ by using the iteration in Eq. (12), Eq. (9) enables the desired damping function $c(\dot{\phi}(t))$ to be determined since the angular velocity $\dot{\phi}(t)$ is assumed to be measured. 

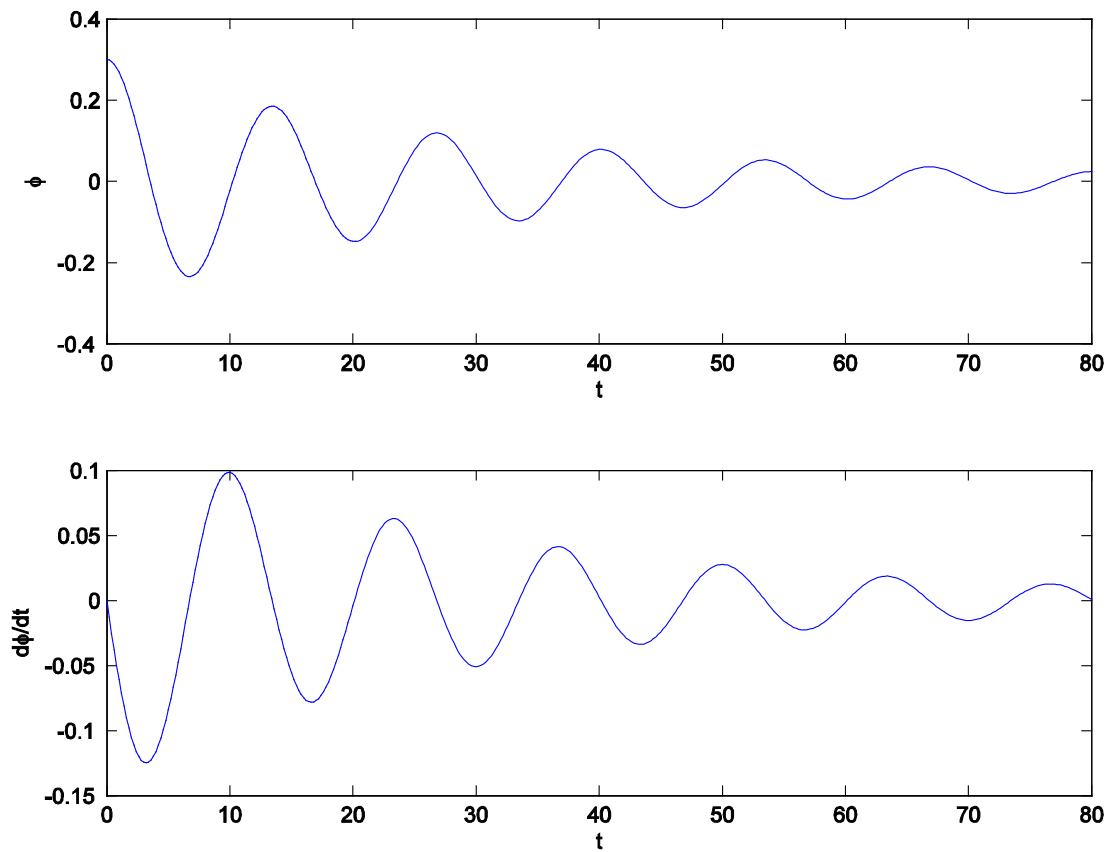

Fig. (1). Time simulations for motion responses of Eq. (15).

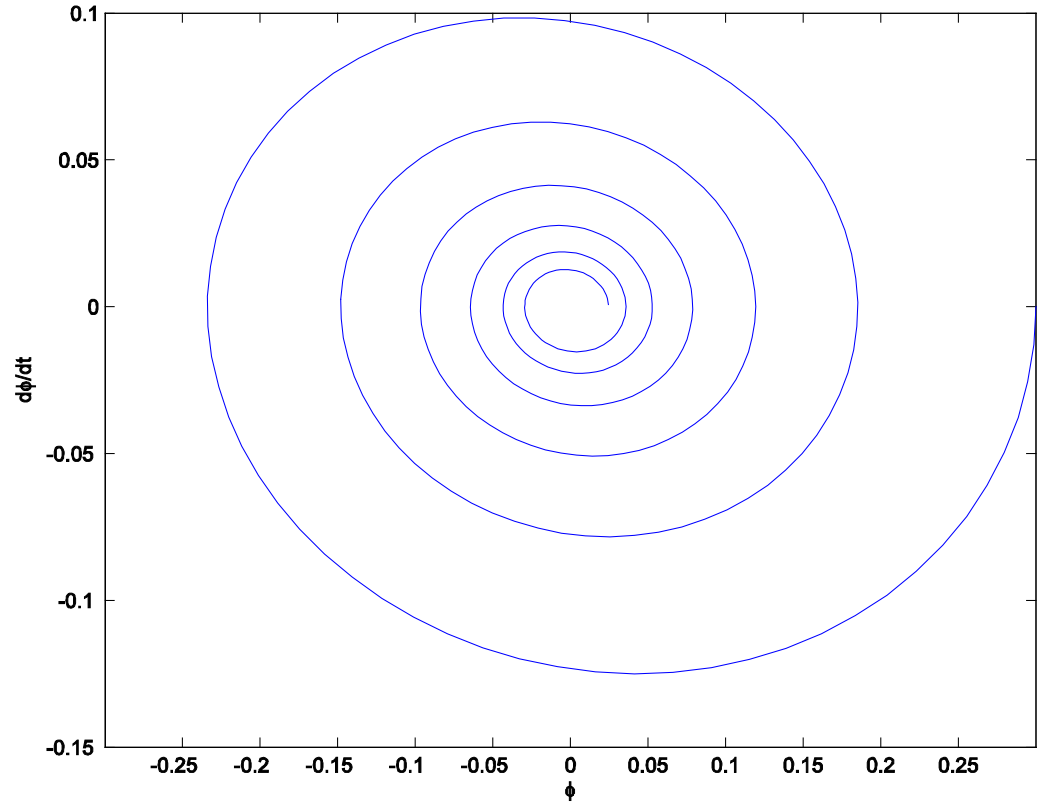

Fig. (2). Phase planes for the model equation (15).

\section{NUMERICAL INVESTIGATIONS: RECOVERING NONLINEAR DAMPING MOMENT OF SHIP}

In this section, we investigate the identification of the nonlinear roll damping moment of a ship by using the suggested method above. For the purpose, we first examine the Gaul disaster $[12,13,15]$ in which the roll motion's equation of ships are modeled as a nonlinear differential equation having a quadratic damping:

$$
\ddot{\phi}+\mu_{1} \dot{\phi}+\mu_{2}|\dot{\phi}| \dot{\phi}+k_{1} \phi+k_{3} \phi^{3}+k_{5} \phi^{5}=0
$$

where $\quad \mu_{1}=0.0555, \mu_{2}=0.1659, \quad k_{1}=0.2227$, $k_{3}=-0.0694, k_{5}=-0.0131$. Comparison of Eqs. (3) and (15) immediately reveals that

$$
\begin{array}{r}
c(\dot{\phi})=\mu_{1} \dot{\phi}+\mu_{2}|\dot{\phi}| \dot{\phi} \text { and } \\
\tilde{k}(\phi)=k_{3} \phi^{3}+k_{5} \phi^{5}
\end{array}
$$

The solution for Eq. (15) can be obtained by integration methods such as Runge-Kutta scheme. Since it is usually convenient to experiment with zero initial velocity, here, we impose the following initial conditions:

$$
\phi(0)=0.3, \dot{\phi}(0)=0
$$

The simulated time solutions and the phase diagram are illustrated in (Figs. 1 and 2), where the results for $\phi(t)$ and $\phi(t)$ are to be considered as measured data for the present inverse study. 


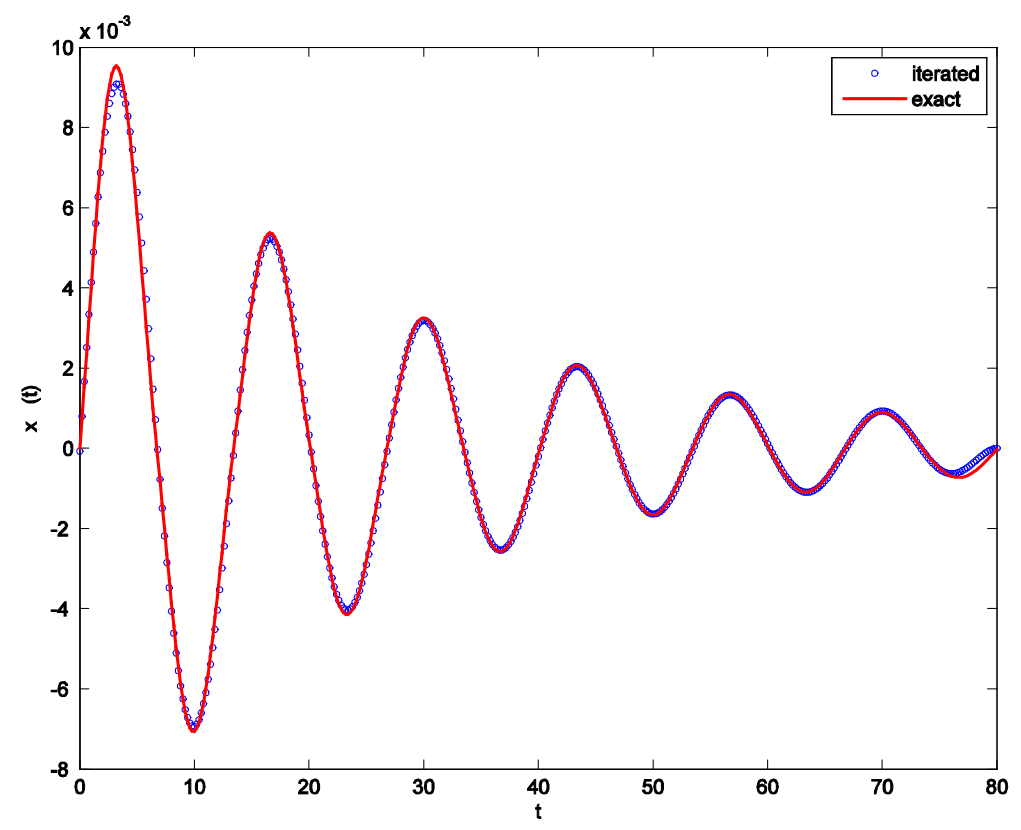

Fig. (3). Comparison of the iterative solution $x_{m}$ with the exact solution $x(t)=-c[\dot{\phi}(t)]$ when $m=10^{3}$.

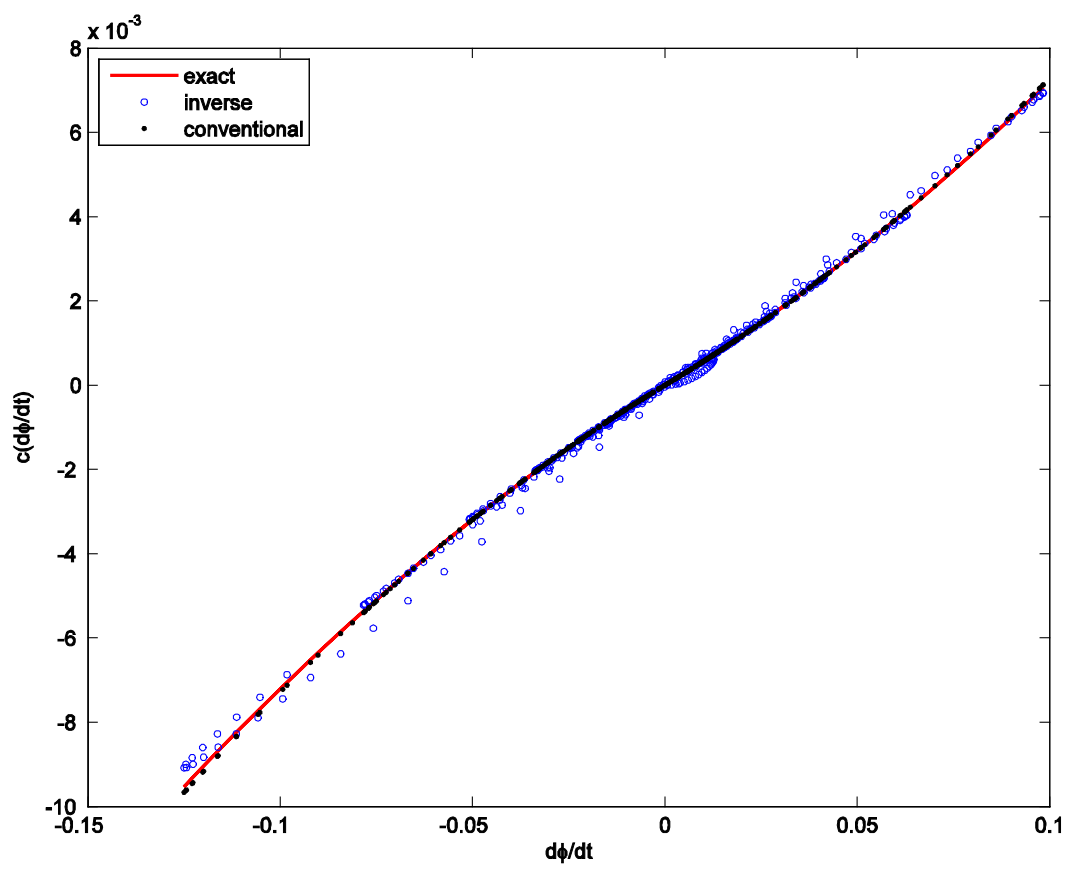

Fig. (4). Illustration of the identified nonlinear damping function compared with the exact function in Eq. (16).

Now, it is time to follow the inverse procedure as described in section 3 for determining the functional form of the nonlinear roll damping function: using the data information of the system's responses $\phi(t)$ and $\dot{\phi}(t)$ as shown in Fig. (1), the ship's nonlinear roll damping $c(\dot{\phi})$ will be determined. This corresponds to solving the integral equation (8).

Conventional numerical treatment does not work for solving Eq. (8) because of the numerical instability (or lack of stability) as discussed in section 3. Thus, we apply the Landweber's regularization method (12) to the integral equation (8) as a stabilization technique. The solutions obtained by using the iteration are depicted in Fig. (3) (when the number of iterations $m=10^{3}$ ). The (iterated) solutions appear to be quite accurate compared with the exact solution.

The nonlinear damping function is then determined by using the relationship in Eq. (9). The result is seen in Fig. (4): the determined damping function is in a good agreement 

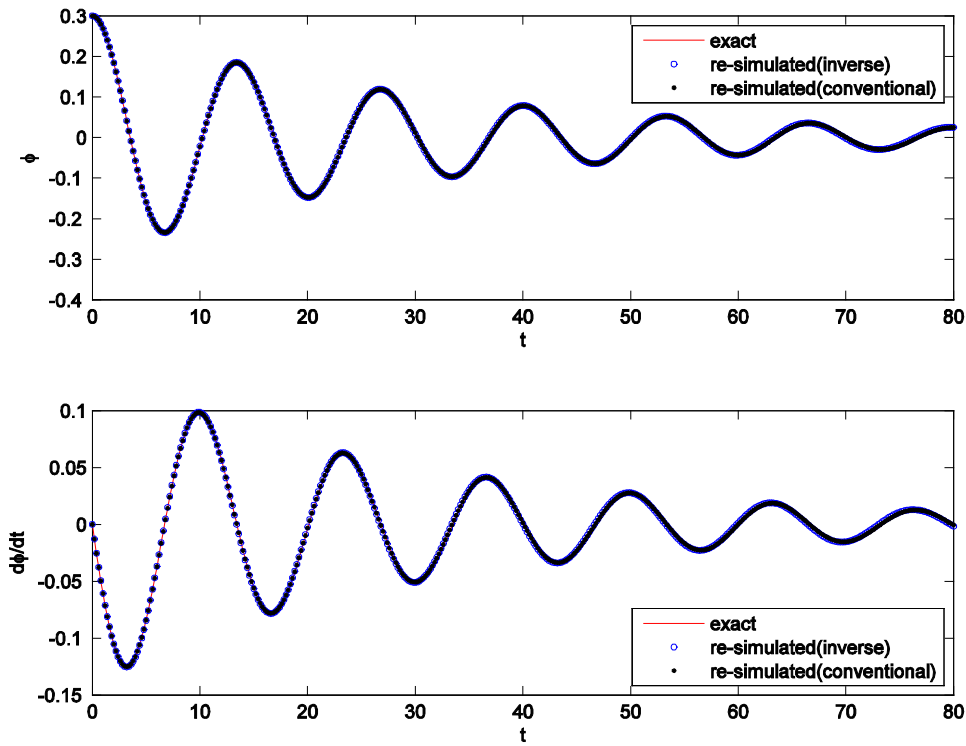

Fig. (5). Re-simulated motion responses by using the identified nonlinear damping function in Fig. (4).
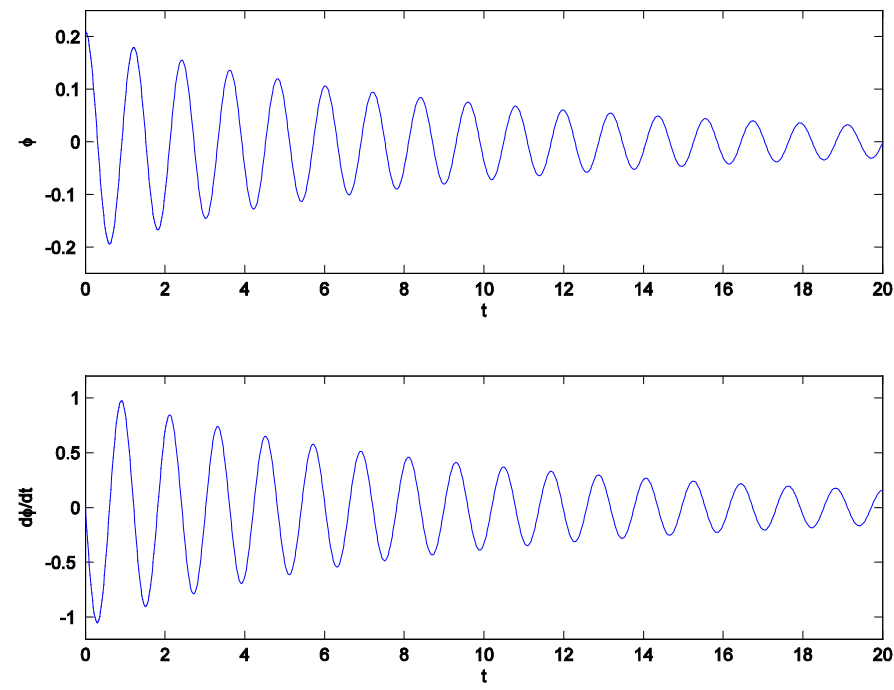

Fig. (6). Simulated motion responses of Eq. (19) for the initial conditions $\phi(0)=0.21$ and $\dot{\phi}(0)=0$.

with the exact one in Eq. (16). In order to verify the rationality and validity of the present method, the comparison is carried out in Fig. (4) with those from a conventional scheme (i.e., parametric identification), which uses the roll decrement data to estimate the coefficient of the prescribed roll model [16]. For the conventional parametric estimation, the nonlinear damping model is assumed to be $v_{1} \dot{\phi}+v_{2}|\dot{\phi}| \dot{\phi}$. It is shown that the result in Fig. (4) is also well coincident with the one from the well-known conventional method. Finally, we re-simulate the roll motion of ship through the angular displacement and angular velocity by using the determined nonlinear damping function in Fig. (4). Fig. (5) shows the roll simulation, which is compared to the exact one and the one from conventional method: it may be hard to find disagreement. This justifies the applicability of the present inverse method to the detecting of the nonlinear roll damping of a ship.
We finish this section with giving another example of cubic nonlinear damping. The model suggested by Wright and Marshfield [1, 14, 15] is

$\ddot{\phi}+\mu_{1} \dot{\phi}+\mu_{3} \dot{\phi}^{3}+\omega_{0}^{2}\left(1-1.402 \phi^{2}+0.271 \phi^{4}\right) \phi=0$

where $\mu_{1}=0.172, \mu_{3}=0.108, \omega_{0}=5.278$. Thus, in this case,

$$
\begin{gathered}
c(\dot{\phi})=\mu_{1} \dot{\phi}+\mu_{3} \dot{\phi}^{3} \text { and } \\
\tilde{k}(\phi)=\omega_{0}{ }^{2}\left(-1.402 \phi^{2}+0.271 \phi^{4}\right) \phi
\end{gathered}
$$

With the initial conditions $\phi(0)=0.21$ and $\dot{\phi}(0)=0$, all the concerning calculations are performed in the same way just above. (Figs. 6 and 7) show the results for the simulated motion responses and phase diagrams of the model equation (19). Through the iteration in Eq. (12), the solution for the integral equation which corresponds to the motion responses 


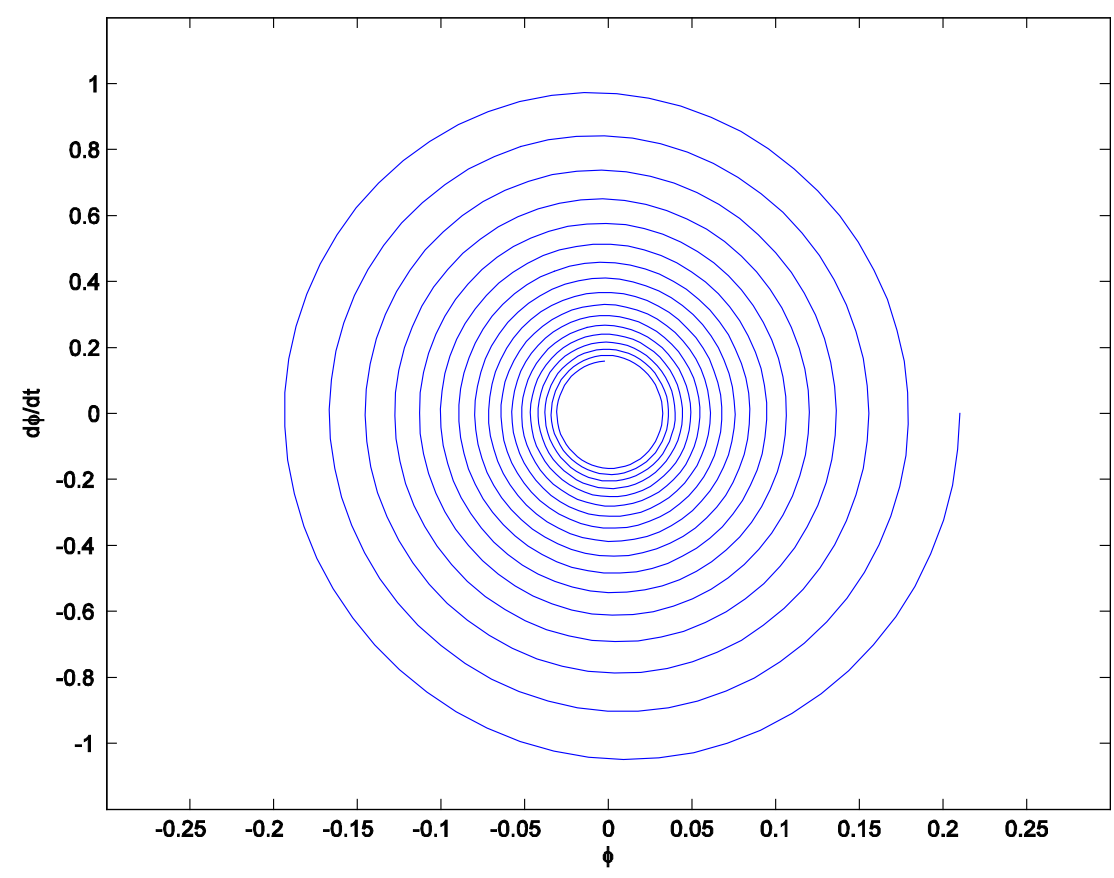

Fig. (7). Phase planes for the model equation (19).

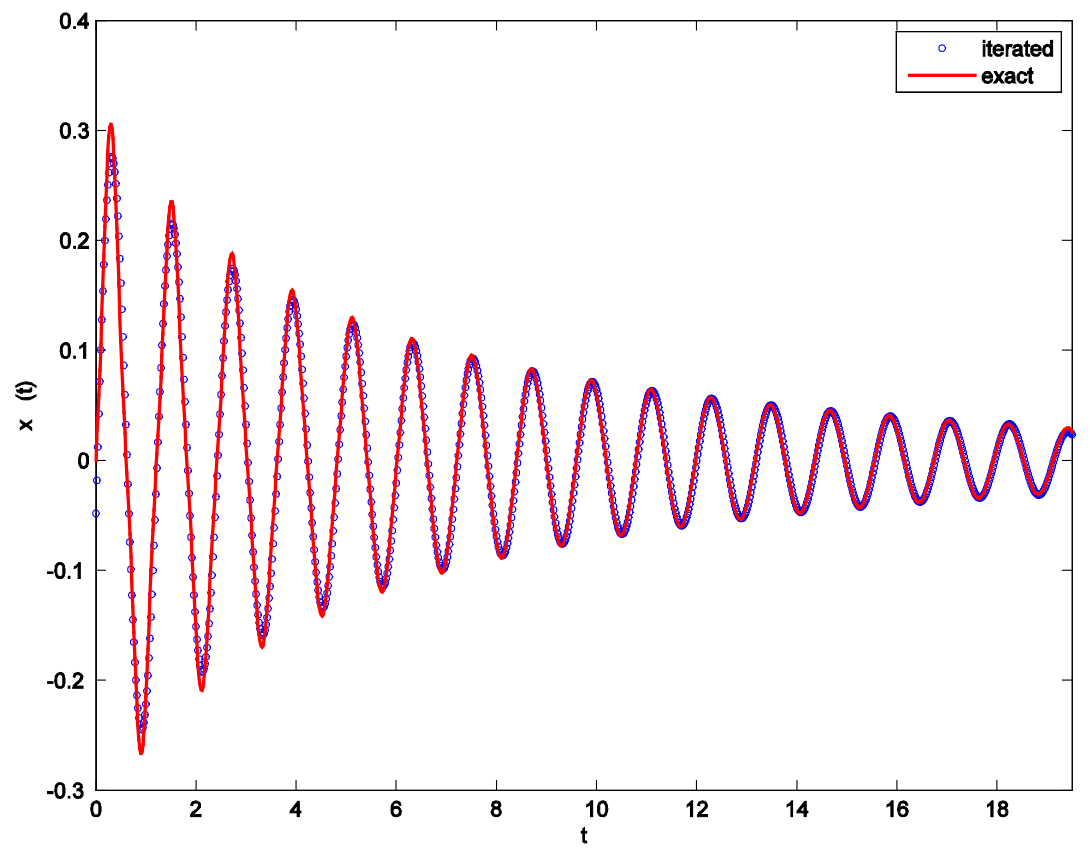

Fig. (8). Comparison of the iterative solution $x_{m}$ with the exact solution $x(t)=-c[\dot{\phi}(t)]$ when $m=10^{3}$.

in Fig. (6) can be obtained: the numerical solution is depicted in Fig. (8) when the number of iterations is $m=10^{3}$. It is clear that the (iterated) solution and the exact solution are in a good agreement. The results in Fig. (8) enable the desired nonlinear damping function to be determined by the same way as illustrated in the first (above) example. The determined nonlinear damping function is shown and compared with the one from the conventional parametric identification method in Fig. (9). Fig. (10) illustrates the resimulated roll responses of ship by using the determined nonlinear damping function: the comparison of the resimulated result is also depicted. In this second example having cubic nonlinear damping, it is concluded that the present inverse method is well justified for detecting the nonlinear roll damping of a ship.

\section{CONCLUSIONS}

This paper treats the determination of nonlinear roll damping moments of ships and ship-shaped floating structures. However, the problem of determination is related with 


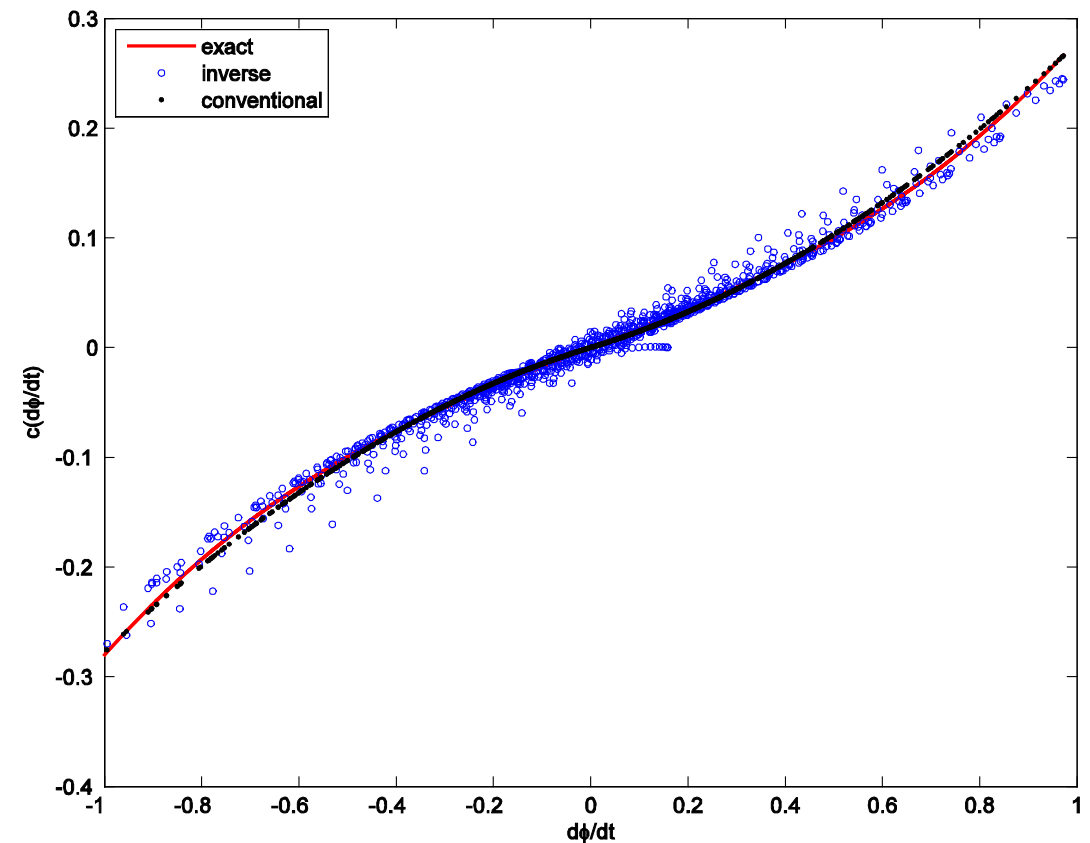

Fig. (9). Illustration of the identified nonlinear damping function compared with the exact function in Eq. (20).
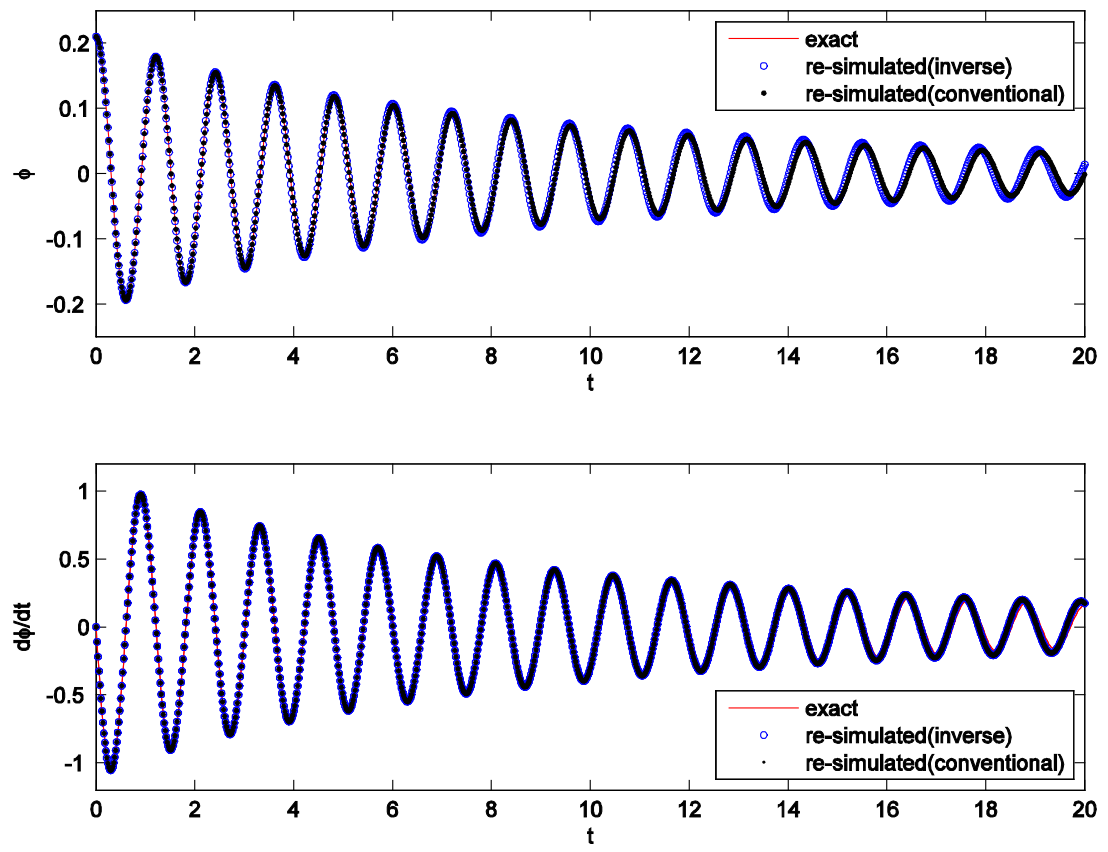

Fig. (10). Re-simulated motion responses by using the identified nonlinear damping function in Fig. (9).

the first kind integral equation, which leads to an ill-posed problem. Thus, its solution lacks stability property. Landweber's regularization is used to tackle the difficulty. Through the regularization process, the functional form of nonlinear damping moments is recovered $[10,11]$. Two different kinds of nonlinear model equations for roll motion are introduced for the numerical investigations: data from the tests $[1,12-$ 15] are used for the numerical study. The present study demonstrates that the method $[10,11]$ can be a good strategy which identifies the nonlinear roll damping moments of ships.

\section{ACKNOWLEDGMENTS}

This work (the first author: Prof. T.S. Jang) was supported by the Mobile Harbor Project of the Korea Advanced Institute of Science and Technology funded by the Ministry of Education, Science and Technology, Korea. The fourth author (Dr. H.G. Sung) acknowledges support from a grant from Ministry of Knowledge and Economics of Korea through the principal R\&D program: Core Methodologies for Design of LNG FSRU's Structure and Mooring System. 


\section{REFERENCES}

[1] J.H.G. Wright and W.B. Marshfield, "Ship roll response and capsize behavior in beam seas", Trans. RINA, vol. 122, pp. 129-146, 1979.

[2] J.O. De Kat and J.R. Paulling, "The simulation of ship motions and capsizing in severe seas". Trans. SNAME, vol. 117, pp. 127-135, 1989.

[3] A. Zborowski and M. Taylan, Evaluation of Small Vessels' Roll Stability Reserve for Resonance Conditions, SNAME Spring Meeting/STAR Symposium, New Orleans, LA. 1989.

[4] M. Taylan, "Application of Nonlinear Roll Motion Model for Assessment of Ship Stability in Waves". Ph.D. Dissertation, Ocean Engineering Department, Florida Institute of Technology, 1990.

[5] M. Taylan, "Nonlinear roll motion of ships in beam waves", Bull. Tech. Univ. Istanbul, vol. 49, pp. 459-479, 1990.

[6] J.A. Witz, C.B. Ablett and J.H. Harrison, "Roll response of semisubmersibles with nonlinear restoring moment characteristics", Appl. Ocean Res., vol. 11, pp. 153-166, 1989.

[7] J.P.F. Denise, "On the roll motion of barges", Transactions RINA, vol. 125 , pp. $255-268,1983$

[8] J.F. Wellicome, "An analytical study of the mechanism of capsizing", International Conference of Stability of Ships and Ocean Vechicles, University of Strathclyde, Glasgow, 1975.

[9] A.O. Odabasi and J. Vince, "Roll response of a ship under the action of a sudden excitation", Int. Shipbuilding Prog., vol. 29, pp. 327-328, 1982.

[10] T.S. Jang, Hang S. Choi and S.L. Han, "A new method for detecting non-linear damping and restoring forces in non-linear oscillation systems from transient data", Int. J. Non-Linear Mech., vol. 44, pp. 801-808, 2009.

[11] T.S. Jang, S.H. Kwon and J.H. Lee, "Recovering the functional form of the nonlinear roll damping of ships from a free-roll decay experiment: an inverse formulism", Ocean Eng. [In press].

[12] A. Morrall, "The Gaul disaster: an investigation into the loss of a large stern trawler", Trans. RINA, vol. 123, pp. 391-416, 1980.

[13] J.B. Cadwell and Y.S. Yang, "Risk and reliability analysis applied to ship capsize: a preliminary study. Proc. Int. Conf. on the SAFESHIP Project: Ship Stability and Safety", Royal Institute of Naval Architects, London, 1986.

[14] A.H. Nayfeh and N.E. Sanchez, "Stability and complicated rolling responses of ships in regular beam seas", Int. Shipbuilding Prog., vol. 37, pp. 331-352, 1990.
[15] H.S.Y. Chan, Z. Xu and W.L. Huang, "Estimation of nonlinear damping coefficients from large-amplitude ship rolling motions", Appl. Ocean Res., vol. 17, pp. 217-224, 1995.

[16] J.F. Dalzell, "A note on the form of ship roll damping", J. Ship Res. vol. 22, pp. 178-185, 1978.

[17] J.A. Murdock, "Perturbations: Theory and Methods," Wiley: New York, 1991.

[18] T.S. Jang, S.H. Kwon and S.L. Han, "A novel method for nonparametric identification of nonlinear restoring forces in nonlinear vibrations from noisy response data: conservative system, nonlinear restoring identification". J. Mech. Sci. Tech., vol. 23, pp. 29382947, 2009.

[19] A. Kirsch, "An Introduction to the Mathematical Theory of Inverse Problems", New York, Springer, 1996.

[20] T.S. Jang and T. Kinoshita, "An ill-posed inverse problem of a wing with locally given velocity data and its analysis", J. Mar. Sci. Technol., vol. 5, pp. 16-20, 2000.

[21] T.S. Jang, H.S. Choi and T. Kinoshita, "Numerical experiments on an ill-posed inverse problem for a given velocity around a hydrofoil by iterative and noniterative regularizations", J. Mar. Sci. Technol., vol. 5, pp. 107-111, 2001.

[22] T.S. Jang, H.S. Choi and T. Kinoshita, "Solution of an unstable inverse problem: wave source evaluation from observation of velocity distribution", J. Mar. Sci. Technol., vol. 6, pp. 181-188, 2001.

[23] T.S. Jang, S.H. Kwon and B.J. Kim, "Solution of an unstable axisymmetric Cauchy-Poisson problem of dispersive water waves for a spectrum with compact support", Ocean Eng., vol. 34, pp. 676684, 2007.

[24] T.S. Jang and S.L. Han, “Application of Tikhonov's regularization to an unstable two dimensional water waves: spectrum with compact support", Ships Offshore Struct., vol. 3, pp. 41-47, 2008.

[25] T.S. Jang, H.G. Sung, S.L. Han and S.H. Kwon, "Inverse determination of the loading source of the infinite beam on elastic foundation”, J. Mech. Sci. Technol., vol. 22, pp. 2350-2356, 2008.

[26] T.S. Jang and S.L. Han, Numerical experiments on determination of spatially concentrated time-varying loads on a beam: an iterative regularization method applied loading identification, J. Mech. Sci. Technol., vol. 23, pp. 2722-2729, 2009.

[27] L. Landweber, "An iteration formula for Fredholm integral equations of the first kind", Am. J. Math., vol. 73, pp. 615-624, 1951.

(c) Jang et al.; Licensee Bentham Open.

This is an open access article licensed under the terms of the Creative Commons Attribution Non-Commercial License (http://creativecommons.org/licenses/by-nc/3.0/) which permits unrestricted, non-commercial use, distribution and reproduction in any medium, provided the work is properly cited. 